\title{
Article
}

\section{L-Fuzzy Sub-Effect Algebras}

\author{
Yan-Yan Dong * and Fu-Gui Shi (D)
}

Beijing Key Laboratory on MCAACI, School of Mathematics and Statistics, Beijing Institute of Technology,

Beijing 102488, China; fuguishi@bit.edu.cn

* Correspondence: 3120195725@bit.edu.cn

\section{check for}

updates

Citation: Dong, Y.-Y.; Shi, F.-G.

L-Fuzzy Sub-Effect Algebras.

Mathematics 2021, 9, 1596. https:/ /

doi.org/10.3390/math9141596

Academic Editor: Sorin Nadaban

Received: 13 May 2021

Accepted: 29 June 2021

Published: 7 July 2021

Publisher's Note: MDPI stays neutral with regard to jurisdictional claims in published maps and institutional affiliations.

Copyright: (C) 2021 by the authors Licensee MDPI, Basel, Switzerland. This article is an open access article distributed under the terms and conditions of the Creative Commons Attribution (CC BY) license (https:// creativecommons.org/licenses/by/ $4.0 /)$.

\begin{abstract}
In this paper, the notions of $L$-fuzzy subalgebra degree and $L$-subalgebras on an effect algebra are introduced and some characterizations are given. We use four kinds of cut sets of $L$-subsets to characterize the $L$-fuzzy subalgebra degree. We induce an $L$-fuzzy convexity by the $L$-fuzzy subalgebra degree, and we prove that a morphism between two effect algebras is an $L$-fuzzy convexity preserving mapping and a monomorphism is an $L$-fuzzy convex-to-convex mapping. Finally, it is proved that the set of all $L$-subalgebras on an effect algebra can form an $L$-convexity, and its $L$-convex hull formula is given.
\end{abstract}

Keywords: effect algebra; $L$-fuzzy subalgebra degree; $L$-subalgebra; $L$-fuzzy convexity; $L$-convex hull formula

\section{Introduction}

Effect algebras were introduced by Foulis and Bennett to axiomatize quantum logic effects on a Hilbert space [1]. The elements of effect algebras represent events that may be unsharp or imprecise. Effect algebras are partial algebras with one partial binary operation that can be converted into bounded posets in general and into lattices in some cases. Since Zadeh introduced the concept of fuzzy sets [2], the theory of fuzzy sets has become a vigorous area of research in different disciplines. In recent years, many scholars have studied (fuzzy) ideals [3,4] and (fuzzy) filters [5,6] (the lattice background is $[0,1]$ ) on effect algebras, but there is no research on the fuzzy subalgebras ( $L$-subalgebras). In order to fill this gap, we first want to extend the unit interval $[0,1]$ to a completely distributive lattice $L$ and introduce the notion of $L$-subalgebras on effect algebras. As we all know, for a given $L$-subset $A, A$ is either an $L$-subalgebra or not, so let us consider the question: what is the degree to which $A$ is an $L$-subalgebra? To solve this question, we propose the concept of $L$-subalgebra degree on effect algebras and investigate their basic properties in this paper.

The notion of convexity [7] is inspired by the shape of some figures, such as circles and polyhedrons in Euclidean spaces. As we all know, a convex structure satisfying the Exchange Law [8] is a matroid, matroids are precisely the structures for which the very simple and efficient greedy algorithm works. Many real-world problems can be defined and solved by making use of matroid theory. So convexity theory has been regarded as an increasingly important role in solving problems. In fact, there exist convexities in many mathematical structures such as semigroup, ring, posets, graphs, convergence spaces and so on [9-13]. It is also natural to consider if there exist convex structures on effect algebras. By these motivations, we will try to prove the existence of convexity on effect algebras.

With the development of fuzzy sets, the notion of convexities has already been extended to fuzzy case. In 1994, Rosa [14] first proposed the notion of fuzzy convex structures with the unit interval [0,1] as the lattice background. In 2009, Y. Maruyama [15] defined another more generalized fuzzy convex structure based on a completely distributive lattice $L$, which is called $L$-convex structure, some of the latest research related to $L$-convex structure can be found in [16-21]. In 2014, a new approach to the fuzzification of convex structures was introduced in [22]. It is called an M-fuzzifying convex structure, in which 
each subset can be regarded as a convex set to some degrees. Further, there are some studies about $M$-fuzzifying convex structures showed in [23-26]. In 2017, abstract convexity was extended to a more general case, which is called an $(L, M)$-fuzzy convex structure in [27]. Particularly, an $(L, L)$-fuzzy structure is briefly called an $L$-fuzzy convex structure. Many researchers investigated $(L, M)$-fuzzy convex structures from different aspects [28-32]. In our paper, we mainly discuss $L$-fuzzy convex structure and $L$-convex structure on an effect algebra.

The paper is organized in the following way. In Section 2, we will give some necessary notations and definitions. In Section 3, we propose the notion of L-fuzzy subalgebra degree on an effect algebra by means of the implication operator of $L$, and we provide their characterizations by cut sets of $L$-subsets. For instance, we give the notion of $L$ subalgebras on effect algebras. In Section 4 , we obtain an $L$-fuzzy convexity induced by $L$ fuzzy subalgebra degree, and we analyze the corresponding $L$-fuzzy convexity preserving mappings and L-fuzzy convex-to-convex mappings. Finally, we prove that the set of all $L$-subalgebras on an effect algebra can form an $L$-convexity, and we provide its $L$-convex hull formula.

\section{Preliminaries}

In this section, we provide some notions and results that will be used in this paper.

Definition 1 ([1]). An effect algebra is a system $(E, \oplus, 0,1)$ consisting of a set $E$ with two special elements $0,1 \in E$, called the zero and the unit, and with a partially defined binary operation $\oplus$ satisfying the following conditions: for any $x, y, z \in E$,

(E1) (Commutative law) if $x \oplus y$ is defined, then $y \oplus x$ is defined and $x \oplus y=y \oplus x$,

(E2) (Associative law) if $x \oplus y$ is defined and $(x \oplus y) \oplus z$ is defined, then $y \oplus z$ and $x \oplus(y \oplus z)$ are defined and $(x \oplus y) \oplus z=x \oplus(y \oplus z)$,

(E3) (Orthosupplement law) for every $x \in E$ there exists a unique $x^{\prime} \in E$ such that $x \oplus x^{\prime}$ is defined and $x \oplus x^{\prime}=1$. The unique element $x^{\prime} \in E$ called the orthosupplement of $x$,

(E4) (Zero-one law) if $x \oplus 1$ is defined, then $x=0$. fied.

In the following, an effect algebra $(E, \oplus, 0,1)$ is denoted by $E$ unless otherwise speci-

Definition 2 ([33]). A nonempty subset $S$ of an effect algebra $E$ is called a subalgebra if it satisfies the following conditions:

(i) $0,1 \in S$,

(ii) $x \in S$ implies $x^{\prime} \in S$,

(iii) if $x, y \in S$ and $x \oplus y$ is defined, then $x \oplus y \in S$.

Let $E$ be an effect algebra. If $x \oplus y$ is defined, then we say $x \perp y$ for all $x, y \in E$. Define a binary relation on $E$ by $x \leq y$ if for some $z \in E, x \oplus z=y$, which turns out to be a partial ordering on $E$ such that 0 and 1 is the smallest element and the greatest element of $E$, respectively. If the poset $(E, \leq)$ is a lattice, then $E$ is called a lattice-ordered effect algebra.

Let $L$ be a complete lattice. We denote the minimal element and the maximal element of $L$ by $\perp_{L}$ and $T_{L}$, respectively. An element $\lambda \in L$ is called co-prime if $\lambda \leq \delta \vee \xi$ implies $\lambda \leq \delta$ or $\lambda \leq \xi$. The set of nonzero co-prime elements in $L$ is denoted by $J(L)$. An element $\lambda \in L$ is called prime if $\lambda \geq \delta \wedge \xi$ implies $\lambda \geq \delta$ or $\lambda \geq \xi$. The set of nonunit prime elements in $L$ is denoted by $P(L)$. From [34], we know that each element of $L$ is the sup of co-prime elements and the inf of prime elements.

Let $\delta, \xi \in L$, the symbol $\delta \prec \xi$ ( $\delta$ is wedge below $\xi$ ) means that for every $H \subseteq L$, $\xi \leq \vee H$ implies the existence of $\eta \in H$ such that $\delta \leq \eta$. A complete lattice $L$ is completely distributive [34] if and only if $\xi=\bigvee\{\delta \mid \delta \prec \xi\}$ for each $\xi \in L$. The set $\{\delta \mid \delta \prec \xi\}$, denoted by $\beta(\xi)$, is called the greatest minimal family of $\xi$ in the sense of [34]. Let $\beta^{*}(\xi)=\beta(\xi) \cap J(L)$. Moreover, define a binary relation $\prec^{o p}$ as follows: for $\xi, \delta \in L$, 
$\xi \prec^{o p} \delta$ if and only if for every subset $H \subseteq L, \wedge H \leq \xi$ implies $\lambda \leq \delta$ for some $\lambda \in H$. The set $\left\{\delta \in L \mid \xi \prec^{o p} \delta\right\}$, denoted by $\alpha(\bar{\xi})$, is the greatest maximal family of $\xi$ in the sense of [34]. Let $\alpha^{*}(\xi)=\alpha(\xi) \cap P(L)$. We know that $\alpha$ is an $\Lambda-\cup$ mapping and $\beta$ is a union-preserving mapping, it holds that $\xi=\vee \beta(\xi)=\vee \beta^{*}(\xi)=\wedge \alpha(\xi)=\wedge \alpha^{*}(\xi)$ for each $\xi \in L$ (see [34]). From [35], we have $\alpha\left(T_{L}\right)=\varnothing$ and $\beta\left(\perp_{L}\right)=\varnothing$.

In the following, a completely distributive lattice $(L, \wedge, \vee)$ is denoted by $L$ unless otherwise specified.

For an effect algebra $E$, each mapping $A: E \longrightarrow L$ is called an $L$-subset of $E$, and we denote the collection of all $L$-subsets of $E$ by $L^{E}$. $L^{E}$ is also a complete lattice by defining " $\leq$ " point-wisely. Furthermore, the smallest element and the largest element in $L^{E}$ are denoted by $\perp$ and $\bar{T}$, respectively. The mapping $g_{L}: L^{E_{1}} \longrightarrow L^{E_{2}}$ is induced by $g: E_{1} \longrightarrow E_{2}$ as follows:

$$
\forall A \in L^{E_{1}}, \forall y \in E_{2}, g_{L}(A)(y)=\bigvee_{g(x)=y} A(x) .
$$

$g_{L}^{\overleftarrow{L}}: L^{E_{2}} \longrightarrow L^{E_{1}}$ is induced by the mapping $g$ as follows:

$$
\forall B \in L^{E_{2}}, \forall x \in E_{1}, g_{L}^{\overleftarrow{L}}(B)(x)=B(g(x))
$$

Definition 3 ([36]). Let $E$ be an effect algebra and $A \in L^{E}$. For any $\lambda \in L$, define

$$
\begin{array}{ll}
A_{[\lambda]}=\{x \in E \mid A(x) \geq \lambda\}, & A^{(\lambda)}=\{x \in E \mid A(x) \not \leq \lambda\}, \\
A_{(\lambda)}=\{x \in E \mid \lambda \in \beta(A(x))\}, & A^{[\lambda]}=\{x \in E \mid \lambda \notin \alpha(A(x))\} .
\end{array}
$$

The right adjoint $\rightarrow$ of the meet operation $\wedge$ is a mapping from $L \times L$ to $L$ defined as

$$
\lambda \rightarrow \mu=\bigvee\{\delta \in L \mid \lambda \wedge \delta \leq \mu\} .
$$

Some basic properties of the operation $\rightarrow$ are listed in the following $[37,38]$.

(1) $\lambda \rightarrow \mu=T_{L} \Leftrightarrow \lambda \leq \mu$;

(2) $\lambda \wedge \delta \leq \mu \Leftrightarrow \lambda \leq \delta \rightarrow \mu$;

(3) $\lambda \leq \delta \Rightarrow \mu \rightarrow \lambda \leq \mu \rightarrow \delta$ and $\lambda \rightarrow \mu \geq \delta \rightarrow \mu$;

(4) $\lambda \rightarrow \bigwedge_{i \in I} \mu_{i}=\bigwedge_{i \in I}\left(\lambda \rightarrow \mu_{i}\right)$.

Let $\perp, \bar{\top} \in L^{E}$ represent $\perp(x)=\perp_{L}$ and $\bar{T}(x)=\top_{L}$ for all $x \in E$. Next, we recall $(L, M)$-fuzzy convexities in [27], which are more general fuzzy convexities. Let $L$ and $M$ be two completely distributive lattices. An $(L, M)$-fuzzy convexity on an effect algebra $E$ is defined as follows:

Definition 4 ([27]). A mapping $\mathcal{C}: L^{E} \longrightarrow M$ is called an $(L, M)$-fuzzy convexity if it satisfies the following conditions:

$(\mathrm{LMC1}) \mathcal{C}(\perp)=\mathcal{C}(\bar{\top})=\top_{M}$;

(LMC2) if $\left\{A_{i} \mid i \in I\right\} \subseteq L^{E}$ is nonempty, then $\mathcal{C}\left(\bigwedge_{i \in I} A_{i}\right) \geq \bigwedge_{i \in I} \mathcal{C}\left(A_{i}\right)$;

(LMC3) if $\left\{A_{i} \mid i \in I\right\} \subseteq L^{E}$ is nonempty and directed, then $\mathcal{C}\left(\vee_{i \in I} A_{i}\right) \geq \wedge_{i \in I} \mathcal{C}\left(A_{i}\right)$.

In this case, the pair $(E, \mathcal{C})$ is called an $(L, M)$-fuzzy convex space. An $(L, L)$-fuzzy convexity is briefly called an L-fuzzy convexity.

An $(L, 2)$-fuzzy convexity is an $L$-convexity in [15]. An $(I, 2)$-fuzzy convexity is a fuzzy convexity in [14], where $I=[0,1]$. A $(2, M)$-fuzzy convexity is an $M$-fuzzifying convexity in [22]. A $(\mathbf{2}, \mathbf{2})$-fuzzy convexity is a convexity in [7].

Definition 5 ([27]). Let $(E, \mathcal{C})$ and $(F, \mathcal{D})$ be two $(L, M)$-fuzzy convex spaces. If $g: E \longrightarrow F$ is a mapping between $E$ and $F$, then

(i) $\quad g:(E, \mathcal{C}) \longrightarrow(F, \mathcal{D})$ is called an $(L, M)$-fuzzy convexity preserving mapping provided that $\mathcal{C}\left(g_{L}^{\leftarrow}(A)\right) \geq \mathcal{D}(A)$ for all $A \in L^{F}$ 
(ii) $\quad g:(E, \mathcal{C}) \longrightarrow(F, \mathcal{D})$ is called an $(L, M)$-fuzzy convex-to-convex mapping provided that $\mathcal{D}\left(g_{L}(B)\right) \geq \mathcal{C}(B)$ for all $B \in L^{E}$.

$A n(L, L)$-fuzzy convexity preserving mapping and an $(L, L)$-fuzzy convex-to-convex mapping are briefly called an L-fuzzy convexity preserving mapping and an L-fuzzy convex-to-convex mapping, respectively.

An $(L, 2)$-fuzzy convexity preserving mapping is an $L$-convexity preserving mapping in [15]. An $(L, 2)$-fuzzy convex-to-convex mapping is an $L$-convex-to-convex mapping in [15].

Definition 6 ([1]). Let $\left\{E_{i}\right\}_{i \in \Lambda}$ be a family of effect algebras, define $\prod_{i \in \Lambda} E_{i}$ as:

$$
\prod_{i \in \Lambda} E_{i}=\left\{x \mid x: \Lambda \longrightarrow \bigcup_{i \in \Lambda} E_{i} \text { s.t. } \forall i \in \Lambda, x_{i}=x(i) \in E_{i}\right\} .
$$

Define the operation $\oplus$ on $\prod_{i \in \Lambda} E_{i}$ as: for $x, y \in \prod_{i \in \Lambda} E_{i}, x \perp y$ iff $x_{i} \perp y_{i}$ for all $i \in \Lambda$. In this case, $(x \oplus y)(i)=x_{i} \oplus y_{i}$ and $x^{\prime}(i)=x_{i}^{\prime}$. Further, $\mathbf{0}_{i}=0_{i}, \mathbf{1}_{i}=1_{i}$ where $0_{i}$ and $1_{i}$ are the minimal element and the maximal element of $E_{i}$. Then $\left(\prod_{i \in \Lambda} E_{i}, \oplus, \mathbf{0}, \mathbf{1}\right)$ is called the direct product of effect algebras.

It is easy to check that $\left(\prod_{i \in \Lambda} E_{i}, \oplus, \mathbf{0}, \mathbf{1}\right)$ is an effect algebra.

Definition 7 ([39]). Let $\left\{E_{i}\right\}_{i=1}^{n}$ be a family of effect algebras and $A_{i}$ be an $L$-subset of $E_{i}$ for all $i \in \Lambda$, then the L-subset $\prod_{i \in \Lambda} A_{i}$ of $\prod_{i \in \Lambda} E_{i}$ is defined by $\left(\prod_{i \in \Lambda} A_{i}\right)(x)=\bigwedge_{i \in \Lambda} A_{i}\left(x_{i}\right)$.

\section{L-Fuzzy Subalgebra Degree on Effect Algebras}

In this section, we introduce the concept of L-fuzzy subalgebra degree on effect algebras by means of the implication operator of $L$. We define an $L$-fuzzy subalgebra provided that its $L$-fuzzy subalgebra degree is equal to $T_{L}$. Moreover, we give some characterizations of $L$-subalgebra degree in terms of four kinds of cut sets of $L$-subsets.

Definition 8. Let $E$ be an effect algebra and $A \in L^{E}$. Then the L-fuzzy subalgebra degree $\mathcal{E}(A)$ of $A$ is defined as:

$$
\mathcal{E}(A)=\bigwedge_{x, y \in E, x \perp y}\left\{[A(x) \rightarrow A(0)] \wedge\left[A(x) \rightarrow A\left(x^{\prime}\right)\right] \wedge[(A(x) \wedge A(y)) \rightarrow A(x \oplus y)]\right\} .
$$

Example 1. Let $E=\{0, x, y, 1\}$ and $\oplus$ be given by:

\begin{tabular}{l|llll}
$\oplus$ & 0 & $x$ & $y$ & 1 \\
\hline 0 & 0 & $x$ & $y$ & 1 \\
$x$ & $x$ & 1 & $*$ & $*$ \\
$y$ & $y$ & $*$ & 1 & $*$ \\
1 & 1 & $*$ & $*$ & $*$
\end{tabular}

Then $(E, \oplus, 0,1)$ is an effect algebra. Let $L=[0,1]$ and define the $L$-subsets of $E$ as follows:

(i) $\quad A_{1}(0)=0.8, A_{1}(x)=0.5, A_{1}(y)=0.5, A_{1}(1)=0.8$. By Definition 8, we can obtain $\mathcal{E}\left(A_{1}\right)=1$.

Indeed, since for any $z \in E, A_{1}(z) \leq A_{1}(0)$, we have $A_{1}(z) \rightarrow A_{1}(0)=1$ for all $z \in E$. We can easily know $x^{\prime}=x, y^{\prime}=y, 0^{\prime}=1$ and $1^{\prime}=0$, it follows from $A_{1}(0)=A_{1}(1)$ that $A_{1}(z)=A_{1}\left(z^{\prime}\right)$, so $A_{1}(z) \rightarrow A_{1}\left(z^{\prime}\right)=1$ for all $z \in E$. We can routinely prove $(A(z) \wedge A(w)) \rightarrow A(z \oplus w)=1$ for all $z, w \in E$ with $z \perp w$. Therefore, $\mathcal{E}\left(A_{1}\right)=1$. 
(ii) $A_{2}(0)=0.3, A_{2}(x)=1, A_{2}(y)=1, A_{2}(1)=1$. By Definition 8, we can obtain $\mathcal{E}\left(A_{2}\right)=$ 0.3 .

Indeed, since $A_{2}(x) \rightarrow A_{2}(0)=A_{2}(1) \rightarrow A_{2}(0)=A_{2}(y) \rightarrow A_{2}(0)=0.3$, we can easily obtain $\mathcal{E}\left(A_{2}\right)=0.3$.

(iii) $A_{3}(0)=0, A_{2}(x)=0.5, A_{3}(y)=1, A_{3}(1)=0.6$. By Definition 8, we can obtain $\mathcal{E}\left(A_{3}\right)=0$.

Indeed, since $A_{3}(y) \rightarrow A_{3}(0)=1 \rightarrow 0=0$, we have $\mathcal{E}\left(A_{3}\right)=0$.

From the properties (3) of the implication operator, the following lemma is obvious.

Lemma 1. Let $E$ be an effect algebra and $A \in L^{E}$. Then $\mathcal{E}(A) \geq \lambda(\lambda \in L)$ if and only if for any $x, y \in E$ satisfying $x \perp y$,

$$
A(x) \wedge A(y) \wedge \lambda \leq A(x \oplus y), A(x) \wedge \lambda \leq A\left(x^{\prime}\right) \text { and } A(x) \wedge \lambda \leq A(0)
$$

By Lemma 1, we can obtain the following theorem.

Theorem 1. Let $E$ be an effect algebra and $A \in L^{E}$. Then

$$
\mathcal{E}(A)=\bigvee\left\{\lambda \in L \mid \forall x, y \in E \text { s.t. } x \perp y, A(x) \wedge A(y) \wedge \lambda \leq A(x \oplus y), A(x) \wedge \lambda \leq A\left(x^{\prime}\right) \wedge A(0)\right\}
$$

We use four kinds of cut sets of $L$-subsets to characterize the $L$-fuzzy subalgebra degree in the following Theorem.

Theorem 2. Let $E$ be an effect algebra and $A \in L^{E}$. Then

(i) $\mathcal{E}(A)=\bigvee\left\{\lambda \in L \mid \forall \mu \leq \lambda, A_{[\mu]}=\varnothing\right.$ or it is a subalgebra of $\left.E\right\}$.

(ii) $\mathcal{E}(A)=\bigvee\left\{\lambda \in L \mid \forall \mu \in P(L), \mu \nsupseteq \lambda, A^{(\mu)}=\varnothing\right.$ or it is a subalgebra of $\left.E\right\}$.

(iii) $\mathcal{E}(A)=\bigvee\left\{\lambda \in L \mid \forall \mu \notin \alpha(\lambda), A^{[\mu]}=\varnothing\right.$ or it is a subalgebra of $\left.E\right\}$.

(iv) $\mathcal{E}(A)=\bigvee\left\{\lambda \in L \mid \forall \mu \in P(L), \mu \notin \alpha(\lambda), A^{[\mu]}=\varnothing\right.$ or it is a subalgebra of $\left.E\right\}$.

(v) $\mathcal{E}(A)=\bigvee\left\{\lambda \in L \mid \forall \mu \in \beta(\lambda), A_{(\mu)}=\varnothing\right.$ or it is a subalgebra of $\left.E\right\}$ if $\beta(\lambda \wedge \mu)=$ $\beta(\lambda) \cap \beta(\mu)$ for all $\lambda, \mu \in L$.

Proof. (i) For any $x, y \in E$ satisfying $x \perp y$, assume that $\lambda \in L$ with the property of $A(x) \wedge A(y) \wedge \lambda \leq A(x \oplus y)$ and $A(x) \wedge \lambda \leq A\left(x^{\prime}\right) \wedge A(0)$. For any $\mu \leq \lambda$, suppose $A_{[\mu]} \neq \varnothing$ and let $x, y \in A_{[\mu]}$ with $x \perp y$, then

$$
\mu=\mu \wedge \lambda \leq A(x) \wedge A(y) \wedge \lambda \leq A(x \oplus y) \text { and } \mu=\mu \wedge \lambda \leq A(x) \wedge \lambda \leq A\left(x^{\prime}\right) \wedge A(0)
$$

which implies $x \oplus y \in A_{[\mu]}$ and $x^{\prime}, 0 \in A_{[\mu]}$. Hence $A_{[\mu]}$ is a subalgebra of $E$. This gives that

$$
\mathcal{E}(A) \leq \bigvee\left\{\lambda \in L \mid \forall \mu \leq \lambda, A_{[\mu]}=\varnothing \text { or it is a subalgebra of } E\right\}
$$

Conversely, assume $\lambda \in L$ and for each $\mu \leq \lambda, A_{[\mu]}=\varnothing$ or it is a subalgebra of $E$. For any $x, y \in E$ satisfying $x \perp y$, let $\mu=A(x) \wedge A(y) \wedge \lambda$ and $\gamma=A(x) \wedge \lambda$, then $\mu \leq \lambda$ and $\gamma \leq \lambda$. It follows that $x, y \in A_{[\mu]}$ and $x \in A_{[\gamma]}$. Since $A_{[\mu]}$ and $A_{[\gamma]}$ are subalgebras of $E$, we have $x \oplus y \in A_{[\mu]}$ and $x^{\prime}, 0 \in A_{[\gamma]}$, i.e., $A(x) \wedge A(y) \wedge \lambda \leq A(x \oplus y)$ and $A(x) \wedge \lambda \leq A\left(x^{\prime}\right) \wedge A(0)$. So we have

$$
\mathcal{E}(A) \geq \bigvee\left\{\lambda \in L \mid \forall \mu \leq \lambda, A_{[\mu]}=\varnothing \text { or it is a subalgebra of } E\right\} .
$$

(ii) For any $x, y \in E$ satisfying $x \perp y$, assume that $\lambda \in L$ with the property of $A(x) \wedge A(y) \wedge \lambda \leq A(x \oplus y)$ and $A(x) \wedge \lambda \leq A\left(x^{\prime}\right) \wedge A(0)$. For any $\mu \in P(L)$ with $\mu \nsupseteq \lambda$, suppose $A^{(\mu)} \neq \varnothing$ and let $x, y \in A^{(\mu)}$ with $x \perp y$, i.e., $A(x) \not \leq \mu$ and $A(y) \not \leq \mu$, then

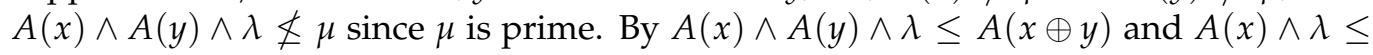


$A\left(x^{\prime}\right) \wedge A(0)$, it follows that $A(x \oplus y) \not \leq \mu$ and $A\left(x^{\prime}\right) \wedge A(0) \not \leq \mu$, i.e., $x \oplus y, x^{\prime}, 0 \in A^{(\mu)}$. Hence $A^{(\mu)}$ is a subalgebra of $E$. This shows that

$$
\mathcal{E}(A) \leq \bigvee\left\{\lambda \in L \mid \forall \mu \in P(L), \mu \nsupseteq \lambda, A^{(\mu)}=\varnothing \text { or it is a subalgebra of } E\right\} .
$$

Conversely, assume that $\lambda \in L$ and $A^{(\mu)}=\varnothing$ or it is a subalgebra of $E$ for $\mu \in P(L)$ with $\mu \nsupseteq \lambda$. For any $x, y \in E$ satisfying $x \perp y$, let $\mu \in P(L)$ and $A(x) \wedge A(y) \wedge \lambda \not \leq \mu$, then $x, y \in A^{(\mu)}$ and $\lambda \not \leq \mu$. Since $A^{(\mu)}$ is a subalgebra of $E$, it holds that $x \oplus y \in A^{(\mu)}$, i.e., $A(x \oplus y) \not \leq \mu$. Hence $A(x) \wedge A(y) \wedge \lambda \leq A(x \oplus y)$. Similarly, we can prove $A(x) \wedge \lambda \leq$ $A\left(x^{\prime}\right) \wedge A(0)$. This shows

$$
\mathcal{E}(A) \geq \bigvee\left\{\lambda \in L \mid \forall \mu \in P(L), \mu \nsupseteq \lambda, A^{(\mu)}=\varnothing \text { or it is a subalgebra of } E\right\} .
$$

(iii) For any $x, y \in E$ satisfying $x \perp y$, assume that $\lambda \in L$ with the property of $A(x) \wedge A(y) \wedge \lambda \leq A(x \oplus y)$ and $A(x) \wedge \lambda \leq A\left(x^{\prime}\right) \wedge A(0)$. For any $\mu \notin \alpha(\lambda)$, suppose $A^{[\mu]} \neq \varnothing$ and let $x, y \in A^{[\mu]}$ with $x \perp y$, i.e., $\mu \notin \alpha(A(x))$ and $\mu \notin \alpha(A(y))$, then by $\mu \notin \alpha(\lambda)$, we have $\mu \notin \alpha(A(x)) \cup \alpha(A(y)) \cup \alpha(\lambda)$. From

$$
\alpha(A(x)) \cup \alpha(A(y)) \cup \alpha(\lambda)=\alpha(A(x) \wedge A(y) \wedge \lambda) \text { and } \alpha(A(x)) \cup \alpha(\lambda)=\alpha(A(x) \wedge \lambda),
$$

we know $\mu \notin \alpha(A(x) \wedge A(y) \wedge \lambda)$ and $\mu \notin \alpha(A(x) \wedge \lambda)$. By $A(x) \wedge A(y) \wedge \lambda \leq A(x \oplus y)$ and $A(x) \wedge \lambda \leq A\left(x^{\prime}\right) \wedge A(0)$, we have $\mu \notin \alpha(A(x \oplus y)), \mu \notin \alpha\left(A\left(x^{\prime}\right)\right)$ and $\mu \notin \alpha(A(0))$, i.e., $x \oplus y, x^{\prime}, 0 \in A^{[\mu]}$. Hence $A^{[\mu]}$ is a subalgebra of $E$. This shows

$$
\mathcal{E}(A) \leq \bigvee\left\{\lambda \in L \mid \forall \mu \notin \alpha(\lambda), A^{[\mu]}=\varnothing \text { or it is a subalgebra of } E\right\} .
$$

Conversely, assume that $\lambda \in L$ and $A^{[\mu]}=\varnothing$ or it is a subalgebra of $E$ for $\mu \notin \alpha(\lambda)$. Now we prove $A(x) \wedge A(y) \wedge \lambda \leq A(x \oplus y)$ and $A(x) \wedge \lambda \leq A\left(x^{\prime}\right) \wedge A(0)$ for all $x, y \in E$ satisfying $x \perp y$. Let $\mu \in L$ and $\mu \notin \alpha(A(x) \wedge A(y) \wedge \lambda)$, then $\mu \notin \alpha(A(x)) \cup \alpha(A(y)) \cup$ $\alpha(\lambda)$. Thus $x, y \in A^{[\mu]}$ and $\mu \notin \alpha(\lambda)$. Since $A^{[\mu]}$ is a subalgebra of $E$, it holds that $x \oplus y \in A^{[\mu]}$, i.e., $\mu \notin \alpha(A(x \oplus y))$. This means $\alpha(A(x) \wedge A(y) \wedge \lambda) \supseteq \alpha(A(x \oplus y))$. Hence

$$
A(x) \wedge A(y) \wedge \lambda=\bigwedge \alpha(A(x) \wedge A(y) \wedge \lambda) \leq \bigwedge \alpha(A(x \oplus y))=A(x \oplus y) .
$$

Similarly, we can prove $A(x) \wedge \lambda \leq A\left(x^{\prime}\right) \wedge A(0)$. This shows

$$
\mathcal{E}(A) \geq \bigvee\left\{\lambda \in L \mid \forall \mu \notin \alpha(\lambda), A^{[\mu]}=\varnothing \text { or it is a subalgebra of } E\right\} .
$$

(iv) By (iii) we first obtain

$$
\mathcal{E}(A) \leq \bigvee\left\{\lambda \in L \mid \forall \mu \in P(L), \mu \notin \alpha(\lambda), A^{[\mu]}=\varnothing \text { or it is a subalgebra of } E\right\}
$$

Conversely, assume that $\lambda \in L$ and $A^{[\mu]}=\varnothing$ or it is a subalgebra of $E$ for all $\mu \in P(L)$ satisfying $\mu \notin \alpha(\lambda)$. Now we prove $A(x) \wedge A(y) \wedge \lambda \leq A(x \oplus y)$ and $A(x) \wedge \lambda \leq A\left(x^{\prime}\right) \wedge$ $A(0)$ for all $x, y \in E$ satisfying $x \perp y$. Let $\mu \in P(L)$ and $\mu \notin \alpha^{*}(A(x) \wedge A(y) \wedge \lambda)$. It follows that

$$
\mu \notin \alpha(A(x) \wedge A(y) \wedge \lambda)=\alpha(A(x)) \cup \alpha(A(y)) \cup \alpha(\lambda) .
$$

Thus $x, y \in A^{[\mu]}$ and $\mu \notin \alpha(\lambda)$. Since $A^{[\mu]}$ is a subalgebra of $E$, it holds that $x \oplus y \in A^{[\mu]}$, i.e., $\mu \notin \alpha(A(x \oplus y))$. So $\mu \notin \alpha^{*}(A(x \oplus y))$, this means $\alpha^{*}(A(x) \wedge A(y) \wedge \lambda) \supseteq \alpha^{*}(A(x \oplus$ $y))$. Hence

$$
A(x) \wedge A(y) \wedge \lambda=\bigwedge \alpha^{*}(A(x) \wedge A(y) \wedge \lambda) \leq \bigwedge \alpha^{*}(A(x \oplus y))=A(x \oplus y) .
$$


Similarly, we can prove $A(x) \wedge \lambda \leq A\left(x^{\prime}\right) \wedge A(0)$. This shows

$$
\mathcal{E}(A) \geq \bigvee\left\{\lambda \in L \mid \forall \mu \in P(L), \mu \notin \alpha(\lambda), A^{[\mu]}=\varnothing \text { or it is a subalgebra of } E\right\}
$$

(v) For any $x, y \in E$ satisfying $x \perp y$, assume that $\perp_{L} \neq \lambda \in L$ with the property of $A(x) \wedge A(y) \wedge \lambda \leq A(x \oplus y)$ and $A(x) \wedge \lambda \leq A\left(x^{\prime}\right) \wedge A(0)$. For any $\mu \in \beta(\lambda)$, suppose $A_{(\mu)} \neq \varnothing$ and let $x, y \in A_{(\mu)}$ with $x \perp y$, i.e., $\mu \in \beta(A(x))$ and $\mu \in \beta(A(y))$. Then by $\mu \in \beta(\lambda)$, we have $\mu \in \beta(A(x)) \cap \beta(A(y)) \cap \beta(\lambda)$. From

$$
\beta(A(x)) \cap \beta(A(y)) \cap \beta(\lambda)=\beta(A(x) \wedge A(y) \wedge \lambda) \text { and } \beta(A(x)) \cap \beta(\lambda)=\beta(A(x) \wedge \lambda),
$$

we know $\mu \in \beta(A(x) \wedge A(y) \wedge \lambda)$ and $\mu \in \beta(A(x) \wedge \lambda)$. By $A(x) \wedge A(y) \wedge \lambda \leq A(x \oplus y)$ and $A(x) \wedge \lambda \leq A\left(x^{\prime}\right) \wedge A(0)$, we have $\mu \in \beta(A(x \oplus y)), \mu \in \beta\left(A\left(x^{\prime}\right)\right)$ and $\mu \in \beta(A(0))$, i.e., $x \oplus y, x^{\prime}, 0 \in A_{(\mu)}$. Hence $A_{(\mu)}$ is a subalgebra of $E$. This shows

$$
\mathcal{E}(A) \leq \bigvee\left\{\lambda \in L \mid \forall \mu \in \beta(\lambda), A_{(\mu)}=\varnothing \text { or it is a subalgebra of } E\right\}
$$

Conversely, assume that $\lambda \in L$ and $A_{(\mu)}=\varnothing$ or it is a subalgebra of $E$ for $\mu \in \beta(\lambda)$. Now we prove $A(x) \wedge A(y) \wedge \lambda \leq A(x \oplus y)$ and $A(x) \wedge \lambda \leq A\left(x^{\prime}\right) \wedge A(0)$ for all $x, y \in E$ satisfying $x \perp y$. The statement holds obviously when $A(x) \wedge A(y) \wedge \lambda=\perp_{L}$. Assume $A(x) \wedge A(y) \wedge \lambda \neq \perp_{L}$, it implies $A(x) \neq \perp_{L}, A(y) \neq \perp_{L}$ and $\lambda \neq \perp_{L}$. Take $\mu \in L$ with $\mu \in \beta(A(x) \wedge A(y) \wedge \lambda)$, then $\mu \in \beta(A(x)) \cap \beta(A(y)) \cap \beta(\lambda)$. Thus $x, y \in A_{(\mu)}$ and $\mu \in \beta(\lambda)$. Since $A_{(\mu)}$ is a subalgebra of $E$, it holds that $x \oplus y \in A_{(\mu)}$, i.e., $\mu \in \beta(A(x \oplus y))$. This means $\beta(A(x) \wedge A(y) \wedge \lambda) \subseteq \beta(A(x \oplus y))$. Hence

$$
A(x) \wedge A(y) \wedge \lambda=\bigvee \beta(A(x) \wedge A(y) \wedge \lambda) \leq \bigvee \beta(A(x \oplus y))=A(x \oplus y)
$$

Similarly, we can prove $A(x) \wedge \lambda \leq A\left(x^{\prime}\right) \wedge A(0)$. This shows

$$
\mathcal{E}(A) \geq \bigvee\left\{\lambda \in L \mid \forall \mu \in \beta(\lambda), A_{(\mu)}=\varnothing \text { or it is a subalgebra of } E\right\}
$$

This completes the proof.

Definition 9. Let $E$ be an effect algebra and $A \in L^{E}$. Then $A$ is called an L-subalgebra provided that $\mathcal{E}(A)=\top_{L}$. In particular, we say an $L$-subalgebra is a fuzzy subalgebra when $L=[0,1]$.

Example 2. In Example 1, it follows from Definition 9 that $A_{1}$ is a fuzzy subalgebra, but $A_{2}$ and $A_{3}$ are not fuzzy subalgebras; $A_{2}$ is a fuzzy subalgebra in the degree of 0.3 .

From Definition 9 and Lemma 1, the following proposition is obvious.

Proposition 1. Let $E$ be an effect algebra and $A \in L^{E}$. Then $A$ is an L-subalgebra if and only if for any $x, y \in E$ with $x \perp y$,

$$
A(0) \geq A(x), A\left(x^{\prime}\right) \geq A(x) \text { and } A(x \oplus y) \geq A(x) \wedge A(y) .
$$

Corollary 1. Let $A$ be an L-subalgebra of an effect algebra $E$. Then

(i) for each $x \in E, A(1) \geq A(x)$;

(ii) for each $x \in E, A(x)=A\left(x^{\prime}\right)$;

(iii) $A(1)=A(0)$.

Proof. (i) Since $x \perp x^{\prime}$ and $A$ is an $L$-subalgebra of $E$, we have

$$
A(1)=A\left(x \oplus x^{\prime}\right) \geq A(x) \wedge A\left(x^{\prime}\right)=A(x) .
$$


(ii) By Proposition 1 we first obtain $A\left(x^{\prime}\right) \geq A(x)$. On the other hand, by Definition 1 (E3), we know $x^{\prime \prime}=x$ and thus $A(x)=A\left(x^{\prime \prime}\right) \geq A\left(x^{\prime}\right)$.

(iii) By (i) we know $A(1) \geq A(0)$. By Proposition 1 , we have $A(1) \leq A(0)$, so $A(1)=A(0)$.

By Definition 9 and Theorem 2, we can obtain the following two results

Corollary 2. Let $A$ be an L-subset of an effect algebra E. Then the following conditions are equivalent:

(i) $A$ is an L-subalgebra;

(ii) for each $\lambda \in J(L), A_{[\lambda]}=\varnothing$ or it is a subalgebra of $E$;

(iii) for each $\lambda \in L, A_{[\lambda]}=\varnothing$ or it is a subalgebra of $E$;

(iv) for each $\lambda \in L, A^{[\lambda]}=\varnothing$ or it is a subalgebra of $E$;

(v) for each $\lambda \in P(L), A^{[\lambda]}=\varnothing$ or it is a subalgebra of $E$;

(vi) for each $\lambda \in P(L), A^{(\lambda)}=\varnothing$ or it is a subalgebra of $E$.

Corollary 3. Let $A$ be an L-subset of an effect algebra E. If $\beta(\lambda \wedge \mu)=\beta(\lambda) \cap \beta(\mu)$ for all $\lambda, \mu \in L$, then the following conditions are equivalent:

(i) $A$ is an L-subalgebra;

(ii) for each $\lambda \in L, A_{(\lambda)}=\varnothing$ or it is a subalgebra of $E$;

(iii) for each $\lambda \in J(L), A_{(\lambda)}=\varnothing$ or it is a subalgebra of $E$.

Let $E$ be an effect algebras and $\left(2^{E}\right)^{L}$ represents the set of all mapping $H: L \longrightarrow 2^{E}$. Then we have the following definitions.

Definition 10 ([36]). Let $H \in\left(2^{E}\right)^{L}$.

(i) If $\mu \in \beta(\lambda)$ implies $H(\lambda) \subset H(\mu)$, then $H$ is called an $L_{\beta}$-nest of $E$.

(ii) If $\mu \in \alpha(\lambda)$ implies $H(\mu) \subset H(\lambda)$, then $H$ is called an $L_{\alpha}$-nest of $E$.

By Theorem 2.3 in [36], we can prove the following two theorems.

Theorem 3. Let $L$ be completely distributive and let $\{A(\lambda) \mid \lambda \in L\})(A(\lambda) \neq \varnothing)$ be an $L_{\alpha}$-nest of subalgebras on an effect algebra $E$. Then there exists an L-subalgebra $B$ such that

(i) $\quad B^{(\lambda)} \subseteq A(\lambda) \subseteq B^{[\lambda]}$ for all $\lambda \in L$;

(ii) $B^{(\lambda)}=\bigcup_{\mu \in \alpha(\lambda)} A(\mu)$ for all $\lambda \in P(L)$;

(iii) $B^{[\mu]}=\bigcap_{\mu \in \alpha(\lambda)} A(\lambda)$ for all $\mu \in P(L)$.

Theorem 4. Let $L$ be completely distributive and let $\{A(\lambda) \mid \lambda \in L\}(A(\lambda) \neq \varnothing)$ be an $L_{\beta}$-nest of subalgebras on an effect algebra $E$. Then there exists an L-subalgebra $B$ such that

(i) $B_{(\lambda)} \subseteq A(\lambda) \subseteq B_{[\lambda]}$ for all $\lambda \in L$;

(ii) $B_{(\lambda)}=\bigcup_{\lambda \in \beta(\mu)} A(\mu)$ for all $\lambda \in L$;

(iii) $B_{[\mu]}=\bigcap_{\lambda \in \beta(\mu)} A(\lambda)$ for all $\mu \in L$.

In particular, when $L=[0,1]$, we have the following result.

Corollary 4. Let $\{A(\lambda) \mid \lambda \in(0,1]\}(A(\lambda) \neq \varnothing)$ be a family of subalgebras on an effect algebra E. If $\lambda<\mu \Rightarrow A(\mu) \subseteq A(\lambda)$, then there exists a fuzzy subalgebra B satisfying

(i) $B_{(\lambda)} \subseteq A(\lambda) \subseteq B_{[\lambda]}$

(ii) $B_{(\lambda)}=\bigcup_{\lambda<\mu} A(\mu)$ for all $\lambda \in(0,1]$;

(iii) $B_{[\mu]}=\bigcap_{\lambda<\mu} A(\lambda)$ for all $\mu \in(0,1]$. 


\section{L-Fuzzy Convexity on Effect Algebras}

In this section, we study the relationship between the $L$-fuzzy subalgebra degree and $L$-fuzzy convexity on an effect algebra. Further, we prove that a morphism between two effect algebras is an L-fuzzy convexity preserving mapping and a monomorphism is an $L$-fuzzy convex-to-convex mapping. Finally, we prove that the set of all $L$-subalgebras on an effect algebra is an $L$-convexity. For instance, we give its $L$-convex hull formula.

For each $A \in L^{E}, \mathcal{E}$ can be naturally considered as a mapping $\mathcal{E}: L^{E} \longrightarrow L$ defined by $A \longmapsto \mathcal{E}(A)$. The following theorem will show that $\mathcal{E}$ is an $L$-fuzzy convexity on an effect algebra $E$.

Theorem 5. Let $E$ be an effect algebra. Then $\mathcal{E}: L^{E} \longrightarrow L$ is an $L$-fuzzy convexity on $E$.

Proof. (LMC1). It is clear that $\mathcal{E}(\perp)=\mathcal{E}(\bar{\top})=T_{L}$ by Definition 8 .

(LMC2). Let $\left\{A_{i}\right\}_{i \in I} \subseteq L^{E}$ be nonempty. Now we show $\mathcal{E}\left(\bigwedge_{i \in I} A_{i}\right) \geq \bigwedge_{i \in I} \mathcal{E}\left(A_{i}\right)$. Let $\lambda \in L$ with $\lambda \leq \wedge_{i \in I} \mathcal{E}\left(A_{i}\right)$. Then for any $i \in I$, we have $\lambda \leq \mathcal{E}\left(A_{i}\right)$, which implies $A_{i}(x) \wedge A_{i}(y) \wedge \lambda \leq A_{i}(x \oplus y)$ and $A_{i}(x) \wedge \lambda \leq A_{i}\left(x^{\prime}\right) \wedge A_{i}(0)$ for all $x, y \in E$ satisfying $x \perp y$. It follows that

$$
\begin{gathered}
\bigwedge_{i \in I} A_{i}(x) \wedge \bigwedge_{i \in I} A_{i}(y) \wedge \lambda \leq \bigwedge_{i \in I} A_{i}(x \oplus y), \\
\bigwedge_{i \in I} A_{i}(x) \wedge \lambda \leq \bigwedge_{i \in I} A_{i}\left(x^{\prime}\right) \wedge \bigwedge_{i \in I} A_{i}(0) .
\end{gathered}
$$

This gives that $\lambda \leq \mathcal{E}\left(\bigwedge_{i \in I} A_{i}\right)$. Hence $\mathcal{E}\left(\bigwedge_{i \in I} A_{i}\right) \geq \bigwedge_{i \in I} \mathcal{E}\left(A_{i}\right)$.

(LMC3) Let $\left\{A_{i}\right\}_{i \in I} \subseteq L^{E}$ be nonempty and directed. Now, we show $\mathcal{E}\left(\bigvee_{i \in I} A_{i}\right) \geq$ $\bigwedge_{i \in I} \mathcal{E}\left(A_{i}\right)$. Let $\lambda \in L$ with $\lambda \leq \bigwedge_{i \in I} \mathcal{E}\left(A_{i}\right)$. Then for any $i \in I, \lambda \leq \mathcal{E}\left(A_{i}\right)$ implies $A_{i}(x) \wedge A_{i}(y) \wedge \lambda \leq A_{i}(x \oplus y)$ and $A_{i}(x) \wedge \lambda \leq A_{i}\left(x^{\prime}\right) \wedge A_{i}(0)$ for all $x, y \in E$ satisfying $x \perp y$. Now, we prove

$$
\bigvee_{i \in I} A_{i}(x) \wedge \bigvee_{i \in I} A_{i}(y) \wedge \lambda \leq \bigvee_{i \in I} A_{i}(x \oplus y)
$$

Take $\mu \in L$ with

$$
\mu \prec \bigvee_{i \in I} A_{i}(x) \wedge \bigvee_{i \in I} A_{i}(y) \wedge \lambda,
$$

then there exists $i, j \in I$ such that $\mu \leq A_{i}(x), \mu \leq A_{j}(y)$ and $\mu \leq \lambda$. Since $\left\{A_{i}\right\}_{i \in I}$ is directed, there exists $i_{0} \in I$ such that $A_{i} \leq A_{i_{0}}$ and $A_{j} \leq A_{i_{0}}$, we thus have $\mu \leq$ $A_{i_{0}}(x) \wedge A_{i_{0}}(y) \wedge \lambda$. By the fact that $A_{i_{0}}(x) \wedge A_{i_{0}}(y) \wedge \lambda \leq A_{i_{0}}(x \oplus y)$, so $\mu \leq A_{i_{0}}(x \oplus y) \leq$ $\bigvee_{i \in I} A_{i}(x \oplus y)$. Hence

$$
\bigvee_{i \in I} A_{i}(x) \wedge \bigvee_{i \in I} A_{i}(y) \wedge \lambda \leq \bigvee_{i \in I} A_{i}(x \oplus y)
$$

Similarly, we can prove

$$
\bigvee_{i \in I} A_{i}(x) \wedge \lambda \leq \bigvee_{i \in I} A_{i}\left(x^{\prime}\right) \wedge \bigvee_{i \in I} A_{i}(0)
$$

Hence $\lambda \leq \mathcal{E}\left(\bigvee_{i \in I} A_{i}\right)$, it implies $\mathcal{E}\left(\bigvee_{i \in I} A_{i}\right) \geq \bigwedge_{i \in I} \mathcal{E}\left(A_{i}\right)$.

Therefore, $\mathcal{E}: L^{E} \longrightarrow L$ is an $L$-fuzzy convexity.

In order to investigate $L$-fuzzy convexity preserving mappings and $L$-fuzzy convexto-convex mappings, we first give following definition and lemma.

Definition 11 ([40]). Let $E$ and $F$ be effect algebras and $g: E \longrightarrow F$ is called 
(i) a morphism if $g\left(1_{E}\right)=1_{F}$, and $x \perp y, x, y \in E$ implies $g(x) \perp g(y)$ in $F$, and $g(x \oplus y)=$ $g(x) \oplus g(y)$;

(ii) a monomorphism if $g$ is a morphism and $g(x) \perp g(y)$ iff $x \perp y$.

Lemma 2. Let $E$ and $F$ be effect algebras and $g: E \longrightarrow F$ is a morphism. Then $g\left(0_{E}\right)=0_{F}$ and $g\left(x^{\prime}\right)=g(x)^{\prime}$ for all $x \in E$

Proof. Since $g\left(0_{E} \oplus 1_{E}\right)=g\left(0_{E}\right) \oplus g\left(1_{E}\right)=g\left(0_{E}\right) \oplus 1_{F}$ by Definition 11, it follows from Definition 1 (E3) that $g\left(0_{E}\right)=0_{F}$. Since $x \perp x^{\prime}$ for any $x \in E$, we have $g\left(1_{E}\right)=g\left(x \oplus x^{\prime}\right)=$ $g(x) \oplus g\left(x^{\prime}\right)$, thus $g\left(x^{\prime}\right)=g(x)^{\prime}$.

Theorem 6. Let $g: E \longrightarrow F$ be a morphism between two effect algebras $E$ and $F$. Then

(i) $g:\left(E, \mathcal{E}_{E}\right) \longrightarrow\left(F, \mathcal{E}_{F}\right)$ is L-fuzzy convexity preserving.

(ii) If $g: E \longrightarrow F$ is a monomorphism, then $g:\left(E, \mathcal{E}_{E}\right) \longrightarrow\left(F, \mathcal{E}_{F}\right)$ is L-fuzzy convex-to-convex.

Proof. (i) In order to prove $g:\left(E, \mathcal{E}_{E}\right) \longrightarrow\left(M, \mathcal{E}_{F}\right)$ is $L$-fuzzy convexity preserving, we just need to prove for any $A \in L^{F}, \mathcal{E}_{E}\left(g_{L}^{\leftarrow}(A)\right) \geq \mathcal{E}_{F}(A)$

Let $\lambda \in L$ with $\lambda \leq \mathcal{E}_{F}(A)$. Then by Lemma 1 we obtain $A(x) \wedge A(y) \wedge \lambda \leq A(x \oplus y)$ and $A(x) \wedge \lambda \leq A\left(x^{\prime}\right) \wedge A(0)$ for all $x, y \in F$ satisfying $x \perp y$. So for any $a, b \in E$ with $a \perp b$,

$$
\begin{aligned}
\lambda \wedge g_{L}^{\leftarrow}(A)(a) \wedge g_{L}^{\leftarrow}(A)(b) & =\lambda \wedge A(g(a)) \wedge A(g(b)) \\
& \leq A(g(a) \oplus g(b)) \\
& =A(g(a \oplus b)) \\
& =g_{L}^{\leftarrow}(A)(a \oplus b)
\end{aligned}
$$

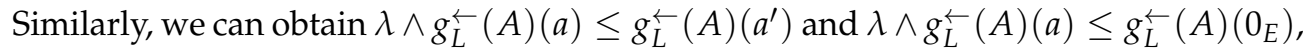

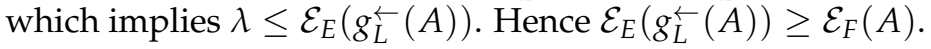

(ii) In order to prove $g:\left(E, \mathcal{E}_{E}\right) \longrightarrow\left(F, \mathcal{E}_{F}\right)$ is $L$-fuzzy convex-to-convex, we just need to prove for any $A \in L^{E}, \mathcal{E}_{E}(A) \leq \mathcal{E}_{F}\left(g_{L}(A)\right)$.

Let $\lambda \in L$ with $\lambda \leq \mathcal{E}_{E}(A)$, then $A(a) \wedge A(b) \wedge \lambda \leq A(a \oplus b)$ and $A(a) \wedge \lambda \leq$ $A\left(a^{\prime}\right) \wedge A(0)$ for all $a, b \in E$ satisfying $a \perp b$. It follows that for $x, y \in F$ satisfying $x \perp y$,

$$
\begin{aligned}
\left(g_{L}(A)\right)(x) \wedge\left(g_{L}(A)\right)(y) \wedge \lambda & =\lambda \wedge \bigvee_{g(a)=x} A(a) \wedge \bigvee_{g(b)=y} A(b) \\
& =\bigvee\{\lambda \wedge A(a) \wedge A(b) \mid g(a)=x, g(b)=y\} \\
& \leq \bigvee\{A(a \oplus b) \mid g(a)=x, g(b)=y\} \\
& \leq \bigvee\{A(a \oplus b) \mid g(a \oplus b)=x \oplus y\} \\
& \leq \bigvee\{A(c) \mid g(c)=x \oplus y\} \\
& =\left(g_{L}(A)\right)(x \oplus y) .
\end{aligned}
$$

Similarly, we can prove $\left(g_{L}(A)\right)(x) \wedge \lambda \leq\left(g_{L}(A)\right)\left(x^{\prime}\right) \wedge\left(g_{L}(A)\right)(0)$. This gives that $\lambda \leq \mathcal{E}_{F}\left(g_{L}(A)\right)$. Hence $\mathcal{E}_{E}(A) \leq \mathcal{E}_{F}\left(g_{L}(A)\right)$. Therefore, $g:\left(E, \mathcal{E}_{E}\right) \longrightarrow\left(F, \mathcal{E}_{F}\right)$ is $L$-fuzzy convex-to-convex.

Corollary 5. Let $\left\{E_{i}\right\}_{i \in \Lambda}$ be a family of effect algebras and $\prod_{i \in \Lambda} A_{i}$ be the direct product of $\left\{A_{i}\right\}_{i \in \Lambda}$, where $A_{i} \in L^{E_{i}}$. If $p_{i}: \prod_{i \in \Lambda} E_{i} \longrightarrow E_{i}$ is the projection, then $p_{i}:\left(\prod_{i \in \Lambda} E_{i}, \mathcal{E}_{i \in \Lambda} E_{i}\right) \longrightarrow\left(E_{i}, \mathcal{E}_{E_{i}}\right)$ is $L-f u z z y$ convexity preserving for all $i \in \Lambda$. 
Proof. It is easy to check that $p_{i}: \prod_{i \in \Lambda} E_{i} \longrightarrow E_{i}$ is a morphism between effects algebras, so by Theorem 6 , we know $p_{i}$ is $L$-fuzzy convexity preserving for all $i \in \Lambda$.

Theorem 7. Let $\left\{E_{i}\right\}_{i \in \Lambda}$ be a family of effect algebras and $\prod_{i \in \Lambda} A_{i}$ be the direct product of $\left\{A_{i}\right\}_{i \in \Lambda}$, where $A_{i} \in L^{E_{i}}$. Then $\mathcal{E}_{i \in \Lambda} E_{i}\left(\prod_{i \in \Lambda} A_{i}\right) \geq \bigwedge_{i \in \Lambda} \mathcal{E}_{E_{i}}\left(A_{i}\right)$.

Proof. Let $p_{i}: \prod_{i \in \Lambda} E_{i} \longrightarrow E_{i}$ be the projection. It can be easily proved that $\prod_{i \in \Lambda} A_{i}=$ $\bigwedge_{i \in \Lambda} p_{i}^{\leftarrow}\left(A_{i}\right)$. So by the proof of (LMC2) in Theorem 8, we have

$$
\mathcal{E}_{i \in \Lambda} E_{i}\left(\prod_{i \in \Lambda} A_{i}\right)=\mathcal{E}_{i \in \Lambda} E_{i}\left(\bigwedge_{i \in \Lambda} p_{i}^{\leftarrow}\left(A_{i}\right)\right) \geq \bigwedge_{i \in \Lambda} \mathcal{E}_{i \in \Lambda} E_{i}\left(p_{i}^{\leftarrow}\left(A_{i}\right)\right) \geq \bigwedge_{i \in \Lambda} \mathcal{E}_{E_{i}}\left(A_{i}\right)
$$

The last inequality holds because $p_{i}$ is $L$-fuzzy convexity preserving by Corollary 4 .

From paper [27], we know $\left(E, \mathcal{E}_{\lambda}\right)$ is an $L$-convex space for all $\lambda \in L \backslash \perp_{L}$. In particular, $\left(E, \mathcal{E}_{T_{L}}\right)$ is an $L$-convex space. We should note that $\mathcal{E}_{T_{L}}=\left\{A \in L^{E} \mid \mathcal{E}(A)=\mathrm{T}_{L}\right\}$, by Definition 9, we obtain that $\mathcal{E}_{T_{L}}$ is the set of all $L$-subalgebras on an effect algebra $E$.

The $L$-convex hull operator with respect to the $L$-convex space $\left(E, \mathcal{E}_{T_{L}}\right)$ is as follows: for $A \in L^{E}, c o(A)=\bigwedge\left\{B \in L^{E} \mid A \leq B \in \mathcal{E}_{\mathrm{T}_{L}}\right\}$ [17], i.e., $c o(A)$ is the least $L$-subalgebra containing $A$. In the following, we will give the corresponding $L$-convex hull formula.

Theorem 8. Let $A$ be an L-subset of an effect algebra $E$. Define for any $x \in E$,

$$
\begin{gathered}
\sigma_{0}=A, \sigma_{1}(x)=\left(\bigvee_{x=x_{1} \oplus x_{2}}\left[A\left(x_{1}\right) \vee A\left(x_{1}^{\prime}\right)\right] \wedge\left[A\left(x_{2}\right) \vee A\left(x_{2}^{\prime}\right)\right]\right) \vee A(x), \\
\text { when } n \geq 2, \sigma_{n}(x)=\bigvee_{x=x_{1} \oplus x_{2}}\left[\sigma_{n-1}\left(x_{1}\right) \vee \sigma_{n-1}\left(x_{1}^{\prime}\right)\right] \wedge\left[\sigma_{n-1}\left(x_{2}\right) \vee \sigma_{n-1}\left(x_{2}^{\prime}\right)\right] .
\end{gathered}
$$

Then $\operatorname{co}(A)=\bigvee_{n=0}^{\infty} \sigma_{n}$.

Proof. We first show $\bigvee_{n=0}^{\infty} \sigma_{n}$ is an $L$-subalgebra of $E$. To achieve this, we give the following statements.

(i). For any $x \in E$ and $n \geq 1, \sigma_{n}(1) \geq \sigma_{n}(x)$.

Let $n=1$. For any $x \in E$, it follows from $x \oplus x^{\prime}=1$ that

$$
\sigma_{1}(1) \geq A(x) \vee A\left(x^{\prime}\right) \geq A(x)=\sigma_{0}(x) .
$$

Let $n \geq 2$. For any $x \in E$, since $x \oplus x^{\prime}=1$, we have that for each $x \in E$.

$$
\begin{aligned}
\sigma_{n}(1) & =\bigvee_{1=y \oplus z}\left[\sigma_{n-1}(y) \vee \sigma_{n-1}\left(y^{\prime}\right)\right] \wedge\left[\sigma_{n-1}(z) \vee \sigma_{n-1}\left(z^{\prime}\right)\right] \\
& \geq\left[\sigma_{n-1}(x) \vee \sigma_{n-1}\left(x^{\prime}\right)\right] \wedge\left[\sigma_{n-1}(x) \vee \sigma_{n-1}\left(x^{\prime}\right)\right] \\
& =\sigma_{n-1}(x) \vee \sigma_{n-1}\left(x^{\prime}\right) .
\end{aligned}
$$

Since for any $x \in E$,

$$
\sigma_{n}(x)=\bigvee_{x=x_{1} \oplus x_{2}}\left[\sigma_{n-1}\left(x_{1}\right) \vee \sigma_{n-1}\left(x_{1}^{\prime}\right)\right] \wedge\left[\sigma_{n-1}\left(x_{2}\right) \vee \sigma_{n-1}\left(x_{2}^{\prime}\right)\right],
$$

then for every pair $\left(x_{1}, x_{2}\right)$ satisfying $x=x_{1} \oplus x_{2}$, it holds that $\sigma_{n-1}\left(x_{1}\right) \vee \sigma_{n-1}\left(x_{1}^{\prime}\right) \leq \sigma_{n}(1)$ and $\sigma_{n-1}\left(x_{2}\right) \vee \sigma_{n-1}\left(x_{2}^{\prime}\right) \leq \sigma_{n}(1)$, we thus obtain $\sigma_{n}(x) \leq \sigma_{n}(1)$.

(ii). $\bigvee_{n=0}^{\infty} \sigma_{n}(x) \leq \bigvee_{n=0}^{\infty} \sigma_{n}(1)$ 
Since $\sigma_{0}=A$ and $1=1 \oplus 0$, we have $\sigma_{1}(1) \geq A(1) \vee A(0) \geq A(1)$. By the above proof, we obtain $\sigma_{1}(1) \geq A(x) \vee A\left(x^{\prime}\right) \geq A(x)$. This implies

$$
\bigvee_{n=0}^{\infty} \sigma_{n}(x)=A(x) \vee \bigvee_{n=1}^{\infty} \sigma_{n}(x) \leq \sigma_{1}(1) \vee \bigvee_{n=1}^{\infty} \sigma_{n}(1) \leq \bigvee_{n=0}^{\infty} \sigma_{n}(1)
$$

(iii). $\sigma_{0}(x) \leq \sigma_{1}(x) \leq \sigma_{2}(x) \leq \cdots$

For any $x \in E$, from the construction of $\sigma_{1}(x)$, it is clear that $\sigma_{1}(x) \geq A(x)=\sigma_{0}(x)$. Let $n \geq 2$. For any $x \in E$, by the fact that $x=x \oplus 0$ and (i), we have

$$
\begin{aligned}
\sigma_{n}(x) & =\bigvee_{x=x_{1} \oplus x_{2}}\left[\sigma_{n-1}\left(x_{1}\right) \vee \sigma_{n-1}\left(x_{1}^{\prime}\right)\right] \wedge\left[\sigma_{n-1}\left(x_{2}\right) \vee \sigma_{n-1}\left(x_{2}^{\prime}\right)\right] \\
& \geq\left[\sigma_{n-1}(x) \vee \sigma_{n-1}\left(x^{\prime}\right)\right] \wedge\left[\sigma_{n-1}(0) \vee \sigma_{n-1}(1)\right] \\
& \geq \sigma_{n-1}(x) .
\end{aligned}
$$

(iv). $\bigvee_{n=0}^{\infty} \sigma_{n}(x) \leq \bigvee_{n=0}^{\infty} \sigma_{n}\left(x^{\prime}\right)$

For any $x \in E$ and $n \geq 2$, since $x^{\prime}=x^{\prime} \oplus 0$, we have

$$
\sigma_{n}\left(x^{\prime}\right) \geq\left[\sigma_{n-1}(x) \vee \sigma_{n-1}\left(x^{\prime}\right)\right] \wedge\left[\sigma_{n-1}(0) \vee \sigma_{n-1}(1)\right] .
$$

By (i) we know that $\sigma_{n-1}(x), \sigma_{n-1}\left(x^{\prime}\right) \leq \sigma_{n-1}(1)$, it follows that $\sigma_{n}\left(x^{\prime}\right) \geq \sigma_{n-1}(x)$. Thus $\bigvee_{n=1}^{\infty} \sigma_{n}(x) \leq \bigvee_{n=2}^{\infty} \sigma_{n}\left(x^{\prime}\right)$. By (iii) we obtain

$$
\bigvee_{n=0}^{\infty} \sigma_{n}(x)=\bigvee_{n=1}^{\infty} \sigma_{n}(x) \leq \bigvee_{n=2}^{\infty} \sigma_{n}\left(x^{\prime}\right)=\bigvee_{n=0}^{\infty} \sigma_{n}\left(x^{\prime}\right)
$$

(v). For $x, y \in E$ and $x \perp y$,

$$
\bigvee_{n=0}^{\infty} \sigma_{n}(x \oplus y) \geq\left(\bigvee_{n=0}^{\infty} \sigma_{n}(x)\right) \wedge\left(\bigvee_{n=0}^{\infty} \sigma_{n}(y)\right)
$$

Let $t \in L$ such that $t \prec\left(\bigvee_{n=0}^{\infty} \sigma_{n}(x)\right) \wedge\left(\bigvee_{n=0}^{\infty} \sigma_{n}(y)\right)$, then we have $t \prec \bigvee_{n=0}^{\infty} \sigma_{n}(x)$ and $t \prec \bigvee_{n=0}^{\infty} \sigma_{n}(y)$. So there exist $i, k \geq 0$ such that $t \leq \sigma_{i}(x)$ and $t \leq \sigma_{j}(y)$. Put $j=\max \{i, k\}$, then by (iii) we obtain $t \leq \sigma_{j}(x)$ and $t \leq \sigma_{j}(y)$. It results that

$$
t \leq \sigma_{j}(x) \wedge \sigma_{j}(y) \leq\left[\sigma_{j}(x) \vee \sigma_{j}\left(x^{\prime}\right)\right] \wedge\left[\sigma_{j}(y) \vee \sigma_{j}\left(y^{\prime}\right)\right] \leq \sigma_{j+1}(x \oplus y) \leq \bigvee_{n=0}^{\infty} \sigma_{n}(x \oplus y)
$$

Hence $\bigvee_{n=0}^{\infty} \sigma_{n}(x \oplus y) \geq\left(\bigvee_{n=0}^{\infty} \sigma_{n}(x)\right) \wedge\left(\bigvee_{n=0}^{\infty} \sigma_{n}(y)\right)$.

(vi). $\bigvee_{n=0}^{\infty} \sigma_{n}(x) \leq \bigvee_{n=0}^{\infty} \sigma_{n}(0)$.

By (ii) and (iv), we have,

$$
\bigvee_{n=0}^{\infty} \sigma_{n}(0)=\bigvee_{n=0}^{\infty} \sigma_{n}\left(1^{\prime}\right) \geq \bigvee_{n=0}^{\infty} \sigma_{n}(1) \geq \bigvee_{n=0}^{\infty} \sigma_{n}(x)
$$

Therefore, by (iii), (iv), (v), (vi) and Proposition 1 , we can obtain that $\bigvee_{n=0}^{\infty} \sigma_{n}$ is an $L$-subalgebra containing $A$. Next, we prove $\bigvee_{n=0}^{\infty} \sigma_{n}$ is the least $L$-subalgebra containing $A$.

Assume $B \in L^{E}$ is an $L$-subalgebra containing $A$, so $A(x) \leq B(x)$ for all $x \in E$. It is clear that $\sigma_{0} \leq B$. Since 


$$
\begin{aligned}
\sigma_{1}(x) & =\left(\bigvee_{x=x_{1} \oplus x_{2}}\left[A\left(x_{1}\right) \vee A\left(x_{1}^{\prime}\right)\right] \wedge\left[A\left(x_{2}\right) \vee A\left(x_{2}^{\prime}\right)\right]\right) \vee A(x) \\
& \leq\left(\bigvee_{x=x_{1} \oplus x_{2}}\left[B\left(x_{1}\right) \vee B\left(x_{1}^{\prime}\right)\right] \wedge\left[B\left(x_{2}\right) \vee B\left(x_{2}^{\prime}\right)\right]\right) \vee B(x) \\
& =\left(\bigvee_{x=x_{1} \oplus x_{2}} B\left(x_{1}\right) \wedge B\left(x_{2}\right)\right) \vee B(x) \quad \text { (by Corrollary } 1 \text { and } B \text { is an L-subalgebra) } \\
& \leq\left(\bigvee_{x=x_{1} \oplus x_{2}} B\left(x_{1} \oplus x_{2}\right)\right) \vee B(x)=B(x) \vee B(x)=B(x) .
\end{aligned}
$$

Assume $\sigma_{k} \leq B(k \geq 2)$ holds. Now,

$$
\begin{aligned}
\sigma_{k+1}(x) & =\bigvee_{x=x_{1} \oplus x_{2}}\left[\sigma_{k}\left(x_{1}\right) \vee \sigma_{k}\left(x_{1}^{\prime}\right)\right] \wedge\left[\sigma_{k}\left(x_{2}\right) \vee \sigma_{k}\left(x_{2}^{\prime}\right)\right] \\
& \leq \bigvee_{x=x_{1} \oplus x_{2}}\left[B\left(x_{1}\right) \vee B\left(x_{1}^{\prime}\right)\right] \wedge\left[B\left(x_{2}\right) \vee B\left(x_{2}^{\prime}\right)\right] \\
& =\bigvee_{x=x_{1} \oplus x_{2}} B\left(x_{1}\right) \wedge B\left(x_{2}\right) \\
& \leq \bigvee_{x=x_{1} \oplus x_{2}} B\left(x_{1} \oplus x_{2}\right)=B(x) .
\end{aligned}
$$

Thus for any $n \geq 0$, it holds that $\sigma_{n} \leq B$, which implies $\bigvee_{n=0}^{\infty} \sigma_{n} \leq B$. This implies $\bigvee_{n=0}^{\infty} \sigma_{n}$ is the least $L$-subalgebra containing $A$. Therefore, $c o(A)=\bigvee_{n=0}^{\infty} \sigma_{n}$.

\section{Conclusions}

In this paper, we proposed the notion of $L$-fuzzy subalgebra degree on an effect algebra based on a completely distributive lattice $L$. We gave their characterizations in terms of four kinds of cut sets of $L$-subsets. We say an $L$-subset is an $L$-subalgebra if its $L$-fuzzy subalgebra degree is equal to $T_{L}$. In fact, the $L$-fuzzy subalgebra degree can describe the degree to which an $L$-subset is an $L$-fuzzy subalgebra. An L-fuzzy convex structure on an effect algebra was naturally constructed and some of its properties were studied. For instance, an $L$-convex structure was induced by the set of all $L$-subalgebras and its $L$-convex hull formula was given.

It should be noted that the same thought can be applied to different algebraic structure such as $M V$-algebras, $B L$-algebras, residuated lattices and so on. Thus, there exist $L$-fuzzy convexities in many logical algebras, which enrich the convexity theory in logical algebras.

Author Contributions: Conceptualization, Y.-Y.D.; formal analysis, Y.-Y.D. and F.-G.S.; funding acquisition, F.-G.S.; investigation, Y.-Y.D. and F.-G.S.; methodology, Y.-Y.D. and F.-G.S.; project administration, Y.-Y.D. and F.-G.S.; supervision, F.-G.S.; validation, Y.-Y.D. and F.-G.S.; visualization, F.-G.S.; writing—original draft, Y.-Y.D.; writing—review and editing, Y.-Y.D. and F.-G.S. Both authors have read and agreed to the published version of the manuscript.

Funding: The project is funded by the National Natural Science Foundation of China (11871097, 12071033), Beijing Institute of Technology Science and Technology Innovation Plan Cultivation Project (No. 2021CX01030) and Chinese Postdoctoral Science Foundation (No. 2020M670142).

Institutional Review Board Statement: Not applicable.

Informed Consent Statement: Not applicable.

Data Availability Statement: Not applicable.

Acknowledgments: The authors express thanks to the handling Editor and the reviewers for their careful reading and useful suggestions.

Conflicts of Interest: The authors declare no conflict of interest. 


\section{References}

1. Foulis, D.J.; Bennett, M.K. Effect algebras and unsharp quantum logics. Found. Phys. 1994, 24, 1331-1352. [CrossRef]

2. Zadeh, L.A. Fuzzy sets. Inf. Control 1965, 8, 338-353. [CrossRef]

3. Ma, Z. Note on ideals of effect algebras. Inf. Sci. 2009, 179, 505-507. [CrossRef]

4. Jenča, G.; Pulmannová, S. Ideals and quotients in lattice ordered effect algebras. Soft Comput. 2001, 5, 376-380. [CrossRef]

5. Foulis, D.J.; Greechie, R.J.; Rüttimann, G.T. Filters and supports in orthoalgebras. Int. J. Theor. Phys. 1992, 31, 789-807. [CrossRef]

6. Liu, D.L.; Wang, G.J. Fuzzy filters in effect algebras. Fuzzy Syst. Math. 2009, 23, 6-16.

7. Berger, M. Convexity. Am. Math. Mon. 1990, 97, 650-678. [CrossRef]

8. Van de Vel, M. Theory of Convex Structures; Elsevier: Amesterdam, The Netherlands, 1993.

9. Fan, C.Z.; Shi, F.-G.; Mehmood, F. M-Hazy Г-Semigroups. J. Nonlinear Convex Anal. 2020, 21, $2659-2669$.

10. Mehmood, F.; Shi, F.-G.; Hayat, K.; Yang, X.P. The homomorphism theorems of M-Hazy rings and their induced fuzzifying convexities. Mathematics 2020, 8, 411. [CrossRef]

11. Mehmood, F.; Shi, F.-G.; Hayat, K. A new approach to the fuzzification of rings. J. Nonlinear Convex Anal. 2020, 21, $2637-2646$.

12. Changat, M.; Mulder, H.M.G.; Sierksma, G. Convexities related to path properties on graphs. Discrete Math. 2005, $290,117-131$. [CrossRef]

13. Pang, B. Categorical properties of $L$-fuzzifying convergence spaces. Filomat 2018, 32, 4021-4036. [CrossRef]

14. Rosa, M.V. On fuzzy topology fuzzy convexity spaces and fuzzy local convexity. Fuzzy Sets Syst. 1994, 62, 97-100. [CrossRef]

15. Maruyama, Y. Lattice-valued fuzzy convex geometry. RIMS Kokyuroku 2009, 164, 22-37.

16. Dong, Y.Y.; Li, J. Fuzzy convex structure and prime fuzzy ideal space on residuated lattices. J. Nonlinear Convex Anal. 2020, 21, 2725-2735.

17. Shen, C.; Shi, F.-G. Characterizations of L-convex spaces via domain theory. Fuzzy Sets Syst. 2020, 380, 44-63. [CrossRef]

18. Wei, X.W.; Wang, B. Fuzzy (restricted) hull operators and fuzzy convex structures on L-sets. J. Nonlinear Convex Anal. 2020, 21, 2805-2815.

19. Li, H.Y.; Wang, K. L-ordered neighborhood systems of stratified L-concave structures. J. Nonlinear Convex Anal. 2020, 21, 2783-2793.

20. Zhang, L.; Pang, B. Strong L-concave structures and L-convergence structures. J. Nonlinear Convex Anal. 2020, $21,2759-2769$.

21. Xiu, Z.-Y. Convergence structures in L-concave spaces. J. Nonlinear Convex Anal. 2020, 21, 2693-2703.

22. Shi, F.-G.; Xiu, Z.-Y. A new approach to the fuzzification of convex structures. J. Appl. Math. 2014, 2014, 1-12. [CrossRef]

23. Pang, B. Convergence structures in M-fuzzifying convex spaces. Quaest. Math. 2020, 43, 1541-1561. [CrossRef]

24. Pang, B. L-fuzzifing convex structures as L-convex structures. J. Nonlinear Convex Anal. 2020, 21, $2831-2841$.

25. Xiu, Z.-Y.; Pang, B. Base axioms and subbase axioms in M-fuzzifying convex spaces. Iran. J. Fuzzy Syst. 2018, $15,75-87$.

26. Zhou, X.-W.; Wang, L. Four kinds of special mappings between M-fuzzifying convex spaces. J. Nonlinear Convex Anal. 2020, 21, 2683-2692.

27. Shi, F.-G.; Xiu, Z.-Y. ( L, M)-fuzzy convex structures. J. Nonlinear Sci. Appl. 2017, 10, 3655-3669. [CrossRef]

28. Li, L.Q. On the category of enriched ( $L, M)$-convex spaces. J. Intell. Fuzzy Syst. 2017, 33, 3209-3216. [CrossRef]

29. Li, J.; Shi, F.-G. L-fuzzy convex induced by L-convex fuzzy sublattice degree. Iran. J. Fuzzy Syst. 2017, 14, 83-102.

30. Pang, B. Bases and subbases in $(L, M)$-fuzzy convex spaces. Comput. Appl. Math. 2020, 39. [CrossRef]

31. Pang, B. Hull operators and interval operators in ( $L, M)$-fuzzy convex spaces. Fuzzy Sets Syst. 2021, 405, 106-127. [CrossRef]

32. Xiu, Z.-Y.; Li, L.; Zhu, Y. A degree approach to special mappings between $(L, M)$-fuzzy convex spaces. J. Nonlinear Convex Anal. 2020, 21, 2625-2635.

33. Dvurečenskij, A.; Pulmannová, S. New Trends in Quantum Structures; Kluwer: Dordrecht, The Netherlands, 2000.

34. Wang, G.J. Theory of topological molecular lattices. Fuzzy Sets Syst. 1992, 47, 351-376.

35. Shi, F.-G. A new approach to the fuzzification of matroids. Fuzzy Sets Syst. 2009, 160, 696-705. [CrossRef]

36. Shi, F.-G. Theory of $L_{\beta}$-nested sets and $L_{\alpha}$-nested sets and application. Fuzzy Syst. Math. 1995, 4, 65-72.

37. War, M.; Dilworth, R.P. Residuated lattices. Trans. Am. Math. Soc. 1939, 45, 335-354.

38. Galatos, N.; Jipsen, P.; Kowalski, T.; Ono, H. Residuated Lattices: An Algebraic Glimpse at Substructural Logics; Elsevier: Amsterdam, The Netherlands, 2007.

39. Shi, F.-G.; Xin, X. L-fuzzy subgroup degrees and L-fuzzy normal subgroup degrees. J. Adv. Res. Pure Math. 2011, 3, 92-108.

40. Jenča, G.; Pulmannová, S. Effect algebras with state operator. Fuzzy Sets Syst. 2015, 260, 43-61. [CrossRef] 